Pacific Journal of Mathematics

TWO EXTREMA PROBLEMS

Marvin Rosenblum and Harold Widow 


\section{TWO EXTREMAL PROBLEMS}

\section{Marvin Rosenblum and HaRold Widom}

1. Introduction. Let $\mathscr{P}_{0}$ be the class of all complex trigonometric polynomials $P$ of the form $P_{0}+P_{1} e^{i \phi}+P_{2} e^{2 i \phi}+\cdots$. Let $\sigma$ and $\mu$ be, respectively normalized Lebesgue measure and any finite non-negative Borel measure on the real interval $(-\pi, \pi]$. Suppose $\mu=\mu_{A}+\mu_{S}$, with $d \mu_{A}(\phi)=f(\phi) d \sigma(\phi)$, is the Lebesgue decomposition of $\mu$ into absolutely continuous and singular measures. In this note we shall be concerned with two generalizations of the problem $Q_{0}$ : Find

$$
I_{0}(\mu)=\inf _{P \in \mathscr{P}_{0}}\left[\int\left|1+e^{i \phi} P\left(e^{i \phi}\right)\right|^{2} d \mu(\phi)\right]^{\frac{1}{2}} .
$$

$Q_{0}$ was solved by Szegö for the case $\mu=\mu_{A}$ and in general by M. G. Krein and Kolmogorov. They showed that $I_{0}(\mu)=\exp \frac{1}{2} \int \log f d \sigma$ if $\log f$ is integrable and $I_{0}(\mu)=0$ otherwise. (See [3], pp. 44, 231.)

We shall consider:

Problem $Q_{1}$ : Suppose $\int|g|^{2} d \mu<\infty$. Find

$$
I_{1}(g, \mu)=\inf _{F \in \mathscr{P}_{0}}\left[\int|g+P|^{2} d \mu\right]^{\frac{1}{2}},
$$

and

Problem $Q_{2}$ : Suppose $\int|h| d \sigma<\infty$. Find

$$
I_{2}(h, \mu)=\sup _{P \in \mathscr{P}_{0}}\left\{\left|\int P h d \sigma\right| /\left[\int|P|^{2} d \mu\right]^{\frac{1}{2}}\right\} .
$$

Clearly $I_{1}\left(e^{-i \phi}, \mu\right)=I_{0}(\mu)$. Also

$$
\left[I_{2}(1, \mu)\right]^{-1}=\inf _{P \in \mathscr{P}_{0}}\left\{\left[\int|P|^{2} d \mu\right]^{\frac{1}{2}} / \int|P d \sigma|\right\}=I_{0}(\mu),
$$

so $Q_{0}$ is a particularization of both $Q_{1}$ and $Q_{2}$. There are other special cases of $Q_{1}$ and $Q_{2}$ that can be found in the work of Szegö [5] and Grenander and Szegö [3]. Of particular interest are the following:

(i ) Let $g(\phi)=e^{-i(k+1) \phi}$, where $k$ is a positive integer. Then $Q_{1}$ is the problem of linear prediction $k$ units ahead of time ([3], p. 184).

(ii) Let $h(\phi)=1 /\left(1-\alpha e^{-i \phi}\right),|\alpha|<1$. Then

$$
I_{2}(h, \mu)=\sup _{P \in \mathscr{P}_{0}}\left\{|P(\alpha)| /\left[\int|P|^{2} d \mu\right]^{\frac{1}{2}}\right\} .
$$

Received October 20, 1959, in revised form February 2, 1960. This work was done while both authors held National Science Foundation postdoctoral fellowships. 
See [3], p. 48.

Throughout we shall indulge in the following notational conveniences: We shall write $I_{1}(g, f)$ and $I_{2}(h, f)$ for $I_{1}\left(g, \mu_{A}\right)$ and $I_{2}\left(h, \mu_{A}\right)$ respectively, and, in certain contexts, consider two functions identical that are equal everywhere except for a set of Lebesgue measure zero.

We have divided this note into six sections. First we indicate an interesting duality between $I_{1}\left(e^{-i \phi} g(\phi), f\right)$ and $I_{2}(g, 1 / f)$ that relates the problems $Q_{1}$ and $Q_{2}$ under certain restrictive hypotheses. In section three we fashion the theory that will handle $Q_{1}$ and $Q_{2}$. This is the solution of a Riemann-Hilbert problem (which we call problem $Q_{3}$ ), which is applied in $\S \S 4,5$ and 6 to $Q_{1}$ and $Q_{2}$.

2. Duality of $I_{1}$ and $I_{2}$. This will fall out of the following Banach space lemma:

Let $\mathscr{P}_{0}$ be a subspace of a Banach space $\mathscr{L}$ and let $\mathscr{P}_{0}^{\perp}$ be the annihilator of $\mathscr{P}_{0}$ in the dual space $\mathscr{L}^{*}$. If $g \in \mathscr{L}$, then

$$
\inf \left\{\|g+P\|: P \in \mathscr{P}_{0}\right\}=\sup \left\{|l(g)|: l \in \mathscr{P}_{0}^{\perp},\|l\| \leqq 1\right\} \text {. }
$$

For a proof see Bonsall [2].

Theorem 1. Suppose $f$ and $1 / f$ are in $L^{1}(-\pi, \pi)$ and $\int|g|^{2} f d \sigma<\infty$. Then

$$
I_{1}\left(e^{-i \phi} g(\phi), f\right)=I_{2}(g, 1 / f) .
$$

Sketch of proof. By the above lemma

$$
I_{1}\left(e^{-i \phi} g(\phi), f\right)=\sup \left\{\left|\int e^{-i \phi} g(\phi) h(\phi) f(\phi) d \sigma\right| /\left[\int|h|^{2} f d \sigma\right]^{\frac{1}{2}}\right\},
$$

where the supremum is taken over all $h$ such that $\int e^{i n \phi} h(\phi) f(\phi) d \sigma=0$ for $n=0,1,2, \cdots$. Through the substitution $e^{-i \phi} h f=P$ if follows that

$$
I_{1}\left(e^{-i \phi} g(\phi), f\right)=\sup \left\{\left|\int P f d \sigma\right| /\left[\int|P|^{2} \frac{1}{f} d \sigma\right]^{\frac{1}{2}}\right\},
$$

where now the supremum is taken over all $P$ such that $\int e^{i n \phi} P(\phi) d \sigma=0$ for $n=1,2, \cdots$. It can be shown that it is sufficient merely to consider suprema for $P \in \mathscr{P}_{0}$, which proves the theorem.

The restrictive condition $1 / f \in L^{1}(-\pi, \pi)$ seems essential to the formulation of the preceding duality relation, but at least this relation indicates that there exist close tie-ins between $Q_{1}$ and $Q_{2}$. We shall solve a Riemann-Hilbert problem for the unit circle that, when applied to $Q_{1}$ and $Q_{2}$, solves both. 
3. The Riemann-Hilbert problem $Q_{3}$. Let $f$ be a non-negative function in $L^{1}=L^{1}(-\pi, \pi)$, and suppose that $\mathscr{P}$ is the closure of $\mathscr{P}_{0}$ in the Hilbert space $L^{2}(f)$ of functions square integrable with respect to the measure $f d \sigma$. Thus, for example, $\mathscr{P}$ in $L^{2}(1)=L^{2}$ can be identified with the Hardy space $H^{2}$. The problem $Q_{3}$ is:

Given $k \in L^{1}$, find functions $P \in \mathscr{P}$ and $q$ satisfying

$$
\begin{gathered}
P f=k+q, \quad \text { and } \\
\int q e^{-i n \phi} d \sigma=0,
\end{gathered}
$$$$
n=0,1, \cdots \text {. }
$$

(Note that since $\int|P|^{2} f d \sigma<\infty$, we have $P f \in L^{1}$ and so $q=P f-k \in L^{1}$.)

We first list some prefactory material. We associate with any nonnegative $f \in L^{1}$ such that $\log f \in L^{1}$ the analytic functions

$$
\begin{aligned}
& F^{+}(z)=\exp \frac{1}{2} \int \frac{e^{i \phi}+z}{e^{i \phi}-z} \log f(\phi) d \sigma(\phi),|z|<1, \\
& F^{-}(z)=\exp \frac{1}{2} \int \frac{z+e^{i \phi}}{z-e^{i \phi}} \log f(\phi) d \sigma(\phi),|z|>1 .
\end{aligned}
$$

$F^{+}$and $F^{-}$belong to $H^{2}$ and $K^{2}$ respectively, and $\overline{F^{-}(z)}=F^{+}(1 / \bar{z})$ if $|z|>1$. (A function $F(z)$ is said to belong to $K^{p}$ if $F(1 / z)$ belongs to $H^{p}$.) Since the boundary functions in $H^{2}$ and $K^{2}$ exist in mean square, we can define

$$
\begin{aligned}
& f^{+}(\phi)=\lim _{r \rightarrow 1-} F^{+}\left(r e^{i \phi}\right), \\
& f^{-}(\phi)=\lim _{r \rightarrow 1+} F^{-}\left(r e^{i \phi}\right) .
\end{aligned}
$$

These functions satisfy

$$
f(\phi)=f^{-}(\phi) f^{+}(\phi)=\left|f^{+}(\phi)\right|^{2}=\left|f^{-}(\phi)\right|^{2} .
$$

For any non-negative $f \in L^{1}$ and $\varepsilon>0$ we define $F_{\varepsilon}^{ \pm}(z), f_{\varepsilon}^{ \pm}(\phi)$ by (3) and (4) with $f$ replaced by $f_{\varepsilon}=f+\varepsilon$. Here we need not assume that $\log f \in L^{1}$. Note that since $f+\varepsilon \geqq \varepsilon>0$, we have $1 / F_{\varepsilon}^{+} \in H^{\infty}$ and $1 / F_{\varepsilon}^{-} \in K^{\infty}$. Moreover $\left|f_{\varepsilon}^{+}(\phi)\right|^{2}=f(\phi)+\varepsilon$, so $\left|f_{\varepsilon}^{-}(\phi)\right|=\left|f_{\varepsilon}^{+}(\phi)\right| \geqq[f(\phi)]^{1 / 2}$.

Next we define an operator ()$_{+}$as follows. Its domain $D$ consists of all $L^{1}$ functions $k$ with Fourier series $\sum_{-\infty}^{\infty} c_{n} e^{i n \phi}$ such that $\sum_{0}^{\infty}\left|c_{n}\right|^{2}<\infty$, and $k_{+}$is the function with Fourier series $\sum_{0}^{\infty} c_{n} e^{i n \phi}$. We define the operator ( ) by $k_{-}=k-k_{+}$. Notice that $k_{+} \in H^{2}$ and $k_{-} \in K^{1}$ with $\int k_{-} d \sigma=0$.

Our discussion of $Q_{3}$ proceeds in the following order. First we prove uniqueness. Then we solve $Q_{3}$ in certain special cases (these being sufficient, it will turn out, to handle $Q_{1}$ ), and finally find the solution in 
the general case.

We are indebted to the referee for the proof of the next lemma.

LEMMA 2. $Q_{3}$ has at most one solution.

Proof. Suppose $P f=q$ where $P \in \mathscr{P}$ and $q$ satisfies (2). Then $P$ is orthogonal, in $L^{2}(f)$, to all exponentials $e^{i n \phi}(n \geqq 0)$. Since $P$ belongs to the closed manifold $\mathscr{P}$ spanned by these exponentials we conclude $P=0$.

One can formally solve $Q_{3}$ by means of the usual factorization methods (see [4], for example). Write $f=f^{+} f^{-}$, so $P f=k+q$ implies

$$
P f^{+}=\frac{k}{f^{-}}+\frac{q}{f^{-}} \text {. }
$$

Applying ()$_{+}$to both sides we obtain $P f^{+}=\left(k / f^{-}\right)_{+}, P=\left(1 / f^{+}\right)\left(k / f^{-}\right)_{+}$. The following theorem justifies this procedure in certain cases.

Theorem 3. ( i ) Suppose $\log f \in L^{1}$ and $k / f^{-} \in D$. Then $Q_{3}$ has the solution

$$
P=\frac{1}{f^{+}}\left(\frac{k}{f^{-}}\right)_{+} \quad q=-f^{-}\left(\frac{k}{f^{-}}\right)_{-} .
$$

(ii) Suppose $\log f \notin L^{1}$ and $k^{2} / f \in L^{1}$. Then $Q_{3}$ has the solution

$$
P=\frac{k}{f} \quad q=0 \text {. }
$$

Proof. (i) Let $\varepsilon>0$. Since the function $f^{+}$is outer, it follows from a theorem of Beurling [1] that there exists a $P_{0} \in \mathscr{P}_{0}$ such that

$$
\int\left|\left(\frac{k}{f^{-}}\right)_{+}-P_{0} f^{+}\right|^{2} d \sigma<\varepsilon
$$

Therefore by (5)

$$
\int\left|\frac{1}{f^{+}}\left(\frac{k}{f^{-}}\right)_{+}-P_{0}\right|^{2} f d \sigma<\varepsilon,
$$

so $P$ as defined in (6) belongs to $\mathscr{P}$. Furthermore, with $q$ as defined in (6),

$$
P f-q=f^{-}\left[\left(\frac{k}{f_{-}}\right)_{+}+\left(\frac{k}{f^{-}}\right)_{-}\right]=k .
$$

It remains to show that $q \in K^{1}$. Certainly $q$ belongs to $K^{1 / 2}$ since it is the product of the two $K^{1}$ functions $-f^{-}$and $\left(k / f^{-}\right)_{-}$. But since also 
$q=P f-k$, it belongs to $L^{1}$. Therefore ([6], p. 163) $q \in K^{1}$.

(ii) If $\log f \notin L^{1}$, the space $\mathscr{P}$ is identical with $L^{2}(f)$ ([3], §33) and so $k / f \in \mathscr{P}$.

We now give the complete solution of $Q_{3}$.

Theorem 4. (i) The limit

$$
\lim _{\varepsilon \rightarrow 0+} \int\left|\left(k / f_{\varepsilon}^{-}\right)_{+}\right|^{2} d \sigma
$$

exists either finitely or infinitely.

(ii) A necessary and sufficient condition that $Q_{3}$ have a solution $P, q$ is that the limit be finite.

(iii) If the limit is finite then

$$
P=\lim \left(1 / f_{\varepsilon}^{+}\right)\left(k / f_{\varepsilon}^{-}\right)_{+}
$$

in the space $L^{2}(f)$, and

$$
\int|P|^{2} f d \sigma=\lim _{\varepsilon \rightarrow 0+} \int\left|\left(k \mid f_{\varepsilon}^{-}\right)_{+}\right|^{2} d \sigma .
$$

Proof. Assume first that $Q_{3}$ has a solution $P, q$ and divide both sides of (1) by $f_{\varepsilon}^{-}$. Since $q / f_{\varepsilon}^{-} \in K^{1}$ and $\int q / f_{\varepsilon}^{-} d \sigma=0$ we have $q / f_{\varepsilon}^{-} \in D$ and $\left(q / f_{\varepsilon}^{-}\right)_{+}=0$; also $P f \mid f_{\varepsilon}^{-} \in L^{2} \subset D$. Therefore we can apply ()$_{+}$to both sides, obtaining

$$
\left(P f \mid f_{\varepsilon}^{-}\right)_{+}=\left(k / f_{\varepsilon}^{-}\right)_{+} \cdot
$$

Consequently

$$
\int\left|\left(k \mid f_{\varepsilon}^{-}\right)_{+}\right|^{2} d \sigma \leqq\left.\int|P f| f_{\varepsilon}^{-}\right|^{2} d \sigma \leqq \int|P|^{2} f d \sigma,
$$

and so

$$
\limsup _{\varepsilon \rightarrow 0+} \int\left|\left(k / f_{\varepsilon}^{-}\right)_{+}\right|^{2} d \sigma<\infty .
$$

Conversely suppose that $\left\{\varepsilon_{n}\right\}$ is a sequence of $\varepsilon$ 's such that $\varepsilon_{n} \rightarrow 0+$ and

$$
\int\left|\left(k / f_{\varepsilon}^{-}\right)_{+}\right|^{2} d \sigma=O(1) \text { for } \varepsilon=\varepsilon_{n} .
$$

By Theorem 3(i) there corresponds to each $\varepsilon=\varepsilon_{n}$ a solution $P_{\varepsilon}, q_{\varepsilon}$ of $(f+\varepsilon) P_{\varepsilon}=k+q_{\varepsilon}$. We have

$$
\int\left|P_{\varepsilon}\right|^{2} f d \sigma \leqq \int\left|P_{\varepsilon}\right|^{2} f_{\varepsilon} d \sigma=\int\left|\left(k / f_{\varepsilon}^{-}\right)_{+}\right|^{2} d \sigma=O(1) \text {. }
$$

Thus there exists a subsequence of $\left\{\varepsilon_{n}\right\}$ such that $\left\{P_{\varepsilon}\right\}$ converges weakly 
in $L^{2}(f)$ to an element $P \in \mathscr{P}$. It will follow that $P, P f-k$ satisfies $Q_{3}$ if the $L^{1}$ function $q=P f-k$ satisfies (2). We have for $n=0,1,2, \cdots$

$$
\begin{aligned}
\int q(\phi) e^{-i n \phi} d \sigma= & \int\left\{P_{\varepsilon}(\phi)[f(\phi)+\varepsilon]-k(\phi)\right\} e^{-i n \phi} d \sigma \\
& +\int\left[P(\phi)-P_{\varepsilon}(\phi)\right] f(\phi) e^{-i n \phi} d \sigma-\varepsilon \int P_{\varepsilon}(\phi) e^{-i n \phi} d \sigma \\
= & J_{1}+J_{2}+J_{3} .
\end{aligned}
$$

Theorem 3(i) implies that $J_{1}=0$. By the weak convergence of the $P_{\varepsilon}$ we can make $J_{2}$ as small as desired by taking $\varepsilon_{n}$ sufficiently small. Finally (10) implies that $\int\left|\varepsilon^{1 / 2} P_{\varepsilon}\right|^{2} d \sigma=O(1)$, so by the Schwarz inequality $\left|J_{3}\right| \leqq \varepsilon^{1 / 2} \int\left|\varepsilon^{1 / 2} P_{\varepsilon}\right| d \sigma=O\left(\varepsilon^{1 / 2}\right)$ as $\varepsilon_{n} \rightarrow 0$. Thus $P, q$ satisfy $Q_{3}$, so (8), holds and (9) is true for any sequence $\left\{\varepsilon_{m}\right\}$ of $\varepsilon$ 's that converge to. $0+$. By what we have shown there corresponds to any such sequence $\left\{\varepsilon_{m}\right\}$ a subsequence such that $P_{\varepsilon}$ converges weakly to the unique (Lemma 2) element $P$. Thus we can consider $\varepsilon$ to be a real variable and conclude that $P_{\varepsilon}$ converges weakly in $L^{2}(f)$ to $P \in \mathscr{P}$ as $\varepsilon \rightarrow 0+$ provided that

$$
\left.\liminf _{\varepsilon \rightarrow 0+} \int \mid k / f_{\varepsilon}^{-}\right)\left._{+}\right|^{2} d \sigma<\infty .
$$

We next prove that in fact $P_{\varepsilon}$ converges strongly to $P$ in $L^{2}(f)$. It suffices to show that $\int\left|P_{\varepsilon}\right|^{2} f d \sigma \rightarrow \int|P|^{2} f d \sigma$. Weak convergence gives

$$
\liminf _{\varepsilon \rightarrow 0+} \int\left|P_{\varepsilon}\right|^{2} f d \sigma \geqq \int|P|^{2} f d \sigma .
$$

On the other hand, as in (7),

$$
\int\left|P_{\varepsilon}\right|^{2} f d \sigma \leqq \int\left|P_{\varepsilon}\right|^{2} f_{\varepsilon} d \sigma=\int\left|\left(k / f_{\varepsilon}^{-}\right)_{+}\right|^{2} d \sigma \leqq \int|P|^{2} f d \sigma .
$$

so

$$
\limsup _{\varepsilon \rightarrow 0+} \int\left|P_{\varepsilon}\right|^{2} f d \sigma \leqq \int|P|^{2} f d \sigma
$$

Thus

$$
\lim _{\varepsilon \rightarrow 0+} \int\left|P_{\varepsilon}\right|^{2} f d \sigma
$$

exists, and equals

$$
\lim _{\varepsilon \rightarrow 0+} \int\left|\left(k / f_{\varepsilon}^{-}\right)_{+}\right|^{2} d \sigma=\int|P|^{2} f d \sigma
$$


Thus the proof is complete.

4. Solution of $Q_{1}$. In $Q_{1}$ we wish to find

$$
I_{1}(g, \mu)=\inf _{P \in \mathscr{P}_{0}}\left[\int|g+P|^{2} d \mu\right]^{\frac{1}{2}},
$$

where $g$ is a given function in $L^{2}(\mu)$. Since $I_{1}(g, \mu)$ represents the distance from $g$ to the manifold $\mathscr{P}_{0}$ in $L^{2}(\mu)$, there exists a (unique) function $P$ belonging to the closure $\mathscr{P}^{\prime}$ of $\mathscr{P}_{0}$ in $L^{2}(\mu)$ such that

$$
I_{1}(g, \mu)=\left[\int|g+P|^{2} d \mu\right]^{\frac{1}{2}} .
$$

This function $P$ is such that $g+P$ is orthogonal to $\mathscr{P}_{0}$, so

$$
\int[g(\phi)+P(\phi)] e^{-i n \phi} d \mu(\phi)=0 \quad n=0,1,2, \cdots .
$$

It follows from a theorem of the brothers Riesz ([6], p. 158) that the measure $\nu$ given by

$$
\nu(E)=\int_{E}[g(\phi)+P(\phi)] d \mu(\phi)
$$

is absolutely continuous with respect to Lebesgue measure. Let $F$ be a Borel set of Lebesgue measure zero such that $\mu_{S}((-\pi, \pi]-F)=0$. Then $g+P$ vanishes on $F$ almost everywhere with respect to $\mu_{s}$, so

$$
\int_{F}|g+P|^{2} d \mu_{S}=0
$$

and

$$
\int|g+P|^{2} d \mu=\int_{\mathscr{C}}|g+P|^{2} d \mu_{A}=\int|g+P|^{2} f d \sigma .
$$

Since $\mu \geqq \mu_{A}$ it follows that $I_{1}(g, \mu)=I_{1}(g, f)$, and this common value is attained by the same extremizing function $P \in \mathscr{P}^{\prime} \subset \mathscr{P}$.

Now,

$$
\int[g(\phi)+P(\phi)] e^{-i n \phi} f(\phi) d \sigma=0 \quad n=0,1, \cdots,
$$

so if we set $q=(g+P) f$ we have $P f=-g f+q$, where $P \in \mathscr{P}$ and $q$ satisfies (2). Since $(g f)^{2} / f=g^{2} f \in L^{1}$, we can apply Theorem 3 to this situation. The extremizing function

$$
P=\left\{\begin{array}{lll}
-\left(1 / f_{+}\right)\left(g f_{+}\right)_{+} & \text {if } & \log f \in L^{1} \\
-g & \text { if } & \log f \notin L^{1},
\end{array}\right.
$$


and since

$$
I_{1}(g, f)=\left[\int|g+P|^{2} f d \sigma\right]^{\frac{1}{2}}=\left[\int|q|^{2} \mid f d \sigma\right]^{\frac{1}{2}}
$$

we have

$$
I_{1}(g, \mu)=I_{1}(g, f)=\left\{\begin{array}{lll}
{\left[\int\left|\left(g f^{+}\right)_{-}\right|^{2} d \sigma\right]^{\frac{1}{2}}} & \text { if } & \log f \in L^{1} \\
0 & \text { if } & \log f \notin L^{1} .
\end{array}\right.
$$

5. Solution of $Q_{2}$. Given $h \in L^{1}$, we will evaluate

$$
I_{2}(h, \mu)=\sup _{P \in \mathscr{F}_{0}}\left\{\left|\int P h d \sigma\right| /\left[\int|P|^{2} d \mu\right]^{\frac{1}{2}}\right\} .
$$

Since $\mu \geqq \mu_{A}$ it is clear that if $I_{2}(h, f)$ is finite so is $I_{2}(h, \mu)$. We shall show that, conversely, if $I_{2}(h, \mu)$ is finite then so is $I_{2}(h, f)$ and in fact $I_{2}(h, f)=I_{2}(h, \mu)$. So now suppose $I_{2}(h, \mu)<\infty$. Then the linear functional $L$ on $\mathscr{P}_{0}$ given by

$$
L(P)=\int P h d \sigma
$$

is bounded on $L^{2}(\mu)$. Therefore if $\mathscr{P}^{\prime}$ denotes the closure of $\mathscr{P}_{0}$ in $L^{2}(\mu)$, there is a uniquely determined $Q \in \mathscr{P}^{\prime}$ such that $L(P)=\int P \bar{Q} d \mu$. Then we have

$$
\int e^{-i n \phi}[Q(\phi) d \mu(\phi)-\bar{h}(\phi) d \sigma(\phi)]=0 \quad n=0,1, \cdots .
$$

We again apply the F. and M. Riesz theorem, and deduce that the measure $\nu$ given by

$$
\nu(E)=\int_{E} Q d \mu-\int_{E} h d \sigma
$$

is absolutely continuous with respect to Lebesgue measure. Letting $F$ be a Borel set of Lebesgue measure zero such that $\mu_{s}((-\pi, \pi]-F)=0$, we see that $Q$ vanishes on $F$ almost everywhere with respect to $\mu_{s}$. Consequently

$$
\int e^{-i n \phi}[Q(\phi) f(\phi)-\bar{h}(\phi)] d \sigma(\phi)=0 \quad n=0,1, \cdots,
$$

so $Q f=\bar{h}+q$, where $Q \in \mathscr{P}^{\prime} \subset \mathscr{P}$ and $q$ satisfies (2). Thus the linear functional

$$
L(P)=\int P h d \sigma=\int P \bar{Q} f d \sigma,
$$


$P \in \mathscr{P}_{0}$, is bounded on $L^{2}(f)$, so $I_{2}(h, f)$ is finite and in fact equals $I_{2}(h, \mu)$. We deduce from Theorem 4 that

$$
I_{2}(h, \mu)=I_{2}(h, f)=\lim _{\varepsilon \rightarrow 0+}\left[\int\left|\left(\bar{h} / f_{\varepsilon}^{-}\right)_{+}\right|^{2} d \sigma\right]^{\frac{1}{2}},
$$

and $Q$ may be exhibited as an $L^{2}(f)$ limit in the mean.

6. Some formulae for $I_{2}(h, \mu)$. We can obtain a simpler formula for $I_{2}(h, \mu)$ if we assume that $h^{2} / f \in L^{1}$ and apply Theorem 3. Then

$$
I_{2}(h, \mu)= \begin{cases}{\left[\int\left|\left(\bar{h} / f^{-}\right)_{+}\right|^{2} d \sigma\right]^{\frac{1}{2}}=\left[\int\left|\left(e^{-i \phi} h(\phi) / f^{+}(\phi)\right)_{-}\right|^{2} d \sigma(\phi)\right]^{\frac{1}{2}}} \\ {\left[\int|h|^{2} / f d \sigma\right]^{\frac{1}{2}}} & \text { if } \log f \in L^{1},\end{cases}
$$

This, in conjunction with our solution of $Q_{1}$, gives the duality discussed in Theorem 1. Note that the hypothesis $1 / f \in L^{1}$ of Theorem 1 implies that $\log f \in L^{1}$.

Another simple formula for $I_{2}(h, \mu)$ is available if we know that the Fourier series $\sum_{-\infty}^{\infty} h_{n} e^{i n \phi}$ of $h$ is such that $h_{-n}=O\left(R_{0}^{-n}\right)$ as $n \rightarrow+\infty$ for some $R_{0}>1$. Then the function $H(z)=\sum_{0}^{\infty} h_{-n} z^{-n}$ is analytic in $|z|>1 / R_{0}$. We have

$$
\int\left|\left(\bar{h} / f_{\varepsilon}^{-}\right)_{+}\right|^{2} d \sigma=\int\left|\left(e^{-i \phi} h(\phi) / f_{\varepsilon}^{+}(\phi)\right)_{-}\right|^{2} d \sigma,
$$

which by the Parseval relation equals

$$
\begin{aligned}
\sum_{n=0}^{\infty}\left|\int e^{i n \phi} h(\phi) f_{\varepsilon}^{+}(\phi) d \sigma\right|^{2} & =\sum_{n=0}^{\infty}\left|\frac{1}{2 \pi} \int_{|z|=1} z^{n+1} H(z) / F_{\varepsilon}^{+}(z) d z\right|^{2} \\
& =\sum_{n=0}^{\infty}\left|\frac{1}{2 \pi} \int_{|z|=R} z^{n+1} H(z) / F_{\varepsilon}^{+}(z) d z\right|^{2},
\end{aligned}
$$

where $1 / R_{0}<R<1$. Let us also assume that $\log f \in L^{1}$, so $F^{+}$is welldefined and

$$
H\left(R e^{i \phi}\right) / F_{\varepsilon}^{+}\left(R e^{i \phi}\right) \longrightarrow H\left(R e^{i \phi}\right) / F^{+}\left(R e^{i \phi}\right)
$$

in $L^{2}$ as $\varepsilon \rightarrow 0+$. It follows that

$$
I_{2}(h, \mu)^{2}=\sum_{n=0}^{\infty}\left|\frac{1}{2 \pi} \int_{|z|=R} z^{n+1} H(z) / F^{+}(z) d z\right|^{2} .
$$

Now, if we write

$$
\frac{1}{F^{+}(z)}=\sum_{n=0}^{\infty} f_{n} z^{n}
$$


then

$$
I_{2}(h, \mu)^{2}=\sum_{n=0}^{\infty}\left|\sum_{m=0}^{\infty} h_{-n-m} f_{m}\right|^{2} .
$$

Thus if $H$ is the Hankel matrix $\left[h_{-n-m}\right]_{n, m=0}^{\infty}$, and $\Phi$ the column vector with components $f_{0}, f_{1}, \cdots$, then

$$
I_{2}(h, \mu)=\|H \Phi\|,
$$

where the norm is that of $l^{2}$.

For example, let $\alpha$ be such that $|\alpha|<1$ and consider

$$
\sup _{P \in \mathscr{P}}\left\{|P(\alpha)| /\left(\int|P|^{2} d \mu\right)^{\frac{1}{2}}\right\} \text {. }
$$

Thus we wish to evaluate $I_{2}\left(1 /\left(1-\alpha e^{-i \phi}\right), \mu\right)$. Here $h_{-n}=\alpha^{n}, n=0,1, \cdots$, so

$$
I_{2}(h, \mu)^{2}=\sum_{n=0}^{\infty}\left|\sum_{m=0}^{\infty} \alpha^{n+m} f_{m}\right|^{2}=1 /\left[\left(1-|\alpha|^{2}\right)\left|F^{+}(\alpha)\right|^{2}\right],
$$

as in [2], p. 48 .

\section{BIBLIOGRAPHY}

1. A. Beurling, On two problems concerning linear transformations in Hilbert space, Acta Math., 81 (1948), 239-255.

2. F. F. Bonsall, Dual extremum problems in the theory of functions, Jour. London Math. Soc., 31 (1956), 1-5-110.

3. U. Grenander and G. Szegö, Toeplitz forms and their applications, Berkeley and Los Angeles, 1958.

4. N. I. Muskhelishvili, Singular integral equations, Groningen, 1953.

5. Szegö, Orthogonal polynomials, A. M. S. colloquium publication, 23 (1939).

6. A. Zygmund, Trigonometrical series, New York, 1952.

INSTITUTE FOR ADVANCED STUDY

UNIVERSITY OF VIRGINIA

CORNELL UNIVERSITY 


\section{PACIFIC JOURNAL OF MATHEMATICS}

\section{EDITORS}

David GILbarg

Stanford University

Stanford, California

\section{F. H. BRowneLL}

University of Washington

Seattle 5, Washington

\section{A. L. Whiteman}

University of Southern California Los Angeles 7, California

\section{J. PAIGe}

University of California

Los Angeles 24, California

\section{ASSOCIATE EDITORS}
E. F. BECKENBACH
T. M. CHERRY
D. DERRY

\author{
E. HEWITT \\ A. HORN \\ L. NACHBIN
}

\author{
M. OHTSUKA \\ H. L. ROYDEN \\ M. M. SCHIFFER
}

E. SPANIER

E. G. STRAUS

F. WOLF

\section{SUPPORTING INSTITUTIONS}

\author{
UNIVERSITY OF BRITISH COLUMBIA \\ CALIFORNIA INSTITUTE OF TECHNOLOGY \\ UNIVERSITY OF CALIFORNIA \\ MONTANA STATE UNIVERSITY \\ UNIVERSITY OF NEVADA \\ NEW MEXICO STATE UNIVERSITY \\ OREGON STATE COLLEGE \\ UNIVERSITY OF OREGON \\ OSAKA UNIVERSITY \\ UNIVERSITY OF SOUTHERN CALIFORNIA
}

\author{
STANFORD UNIVERSITY \\ UNIVERSITY OF TOKYO \\ UNIVERSITY OF UTAH \\ WASHINGTON STATE COLLEGE \\ UNIVERSITY OF WASHINGTON \\ AMERICAN MATHEMATICAL SOCIETY \\ CALIFORNIA RESEARCH CORPORATION \\ HUGHES AIRCRAFT COMPANY \\ SPACE TECHNOLOGY LABORATORIES \\ NAVAL ORDNANCE TEST STATION
}

\footnotetext{
Mathematical papers intended for publication in the Pacific Journal of Mathematics should be typewritten (double spaced), and the author should keep a complete copy. Manuscripts may be sent to any one of the four editors. All other communications to the editors should be addressed to the managing editor, L. J. Paige at the University of California, Los Angeles 24, California.

50 reprints per author of each article are furnished free of charge; additional copies may be obtained at cost in multiples of 50 .
}

The Pacific Journal of Mathematics is published quarterly, in March, June, September, and December. The price per volume (4 numbers) is $\$ 12.00$; single issues, $\$ 3.50$. Back numbers are available. Special price to individual faculty members of supporting institutions and to individual members of the American Mathematical Society: $\$ 4.00$ per volume; single issues, $\$ 1.25$.

Subscriptions, orders for back numbers, and changes of address should be sent to Pacific Journal of Mathematics, 2120 Oxford Street, Berkeley 4, California.

Printed at Kokusai Bunken Insatsusha (International Academic Printing Co., Ltd.), No. 6, 2-chome, Fujimi-cho, Chiyoda-ku, Tokyo, Japan.

PUBLISHED BY PACIFIC JOURNAL OF MATHEMATICS, A NON-PROFIT CORPORATION

The Supporting Institutions listed above contribute to the cost of publication of this Journal, but they are not owners or publishers and have no responsibility for its content or policies. 


\section{Pacific Journal of Mathematics}

\section{Vol. 10, No. $4 \quad$ December, 1960}

M. Altman, An optimum cubically convergent iterative method of inverting a linear bounded operator in Hilbert space . . . . . . . . . . . . . . . . . . . . . . . . . . 1107

Nesmith Cornett Ankeny, Criterion for rth power residuacity ................. 1115

Julius Rubin Blum and David Lee Hanson, On invariant probability measures I . . . . . 1125

Frank Featherstone Bonsall, Positive operators compact in an auxiliary topology ..... 1131

Billy Joe Boyer, Summability of derived conjugate series . . . . . . . . . . . . . . . . 1139

Delmar L. Boyer, A note on a problem of Fuchs . . . . . . . . . . . . . . . . . 1147

Hans-Joachim Bremermann, The envelopes of holomorphy of tube domains in infinite

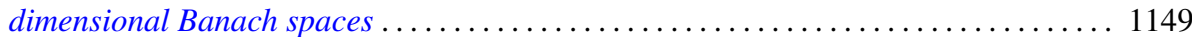

Andrew Michael Bruckner, Minimal superadditive extensions of superadditive

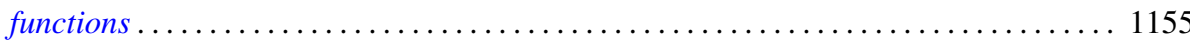

Billy Finney Bryant, On expansive homeomorphisms .................... 1163

Jean W. Butler, On complete and independent sets of operations in finite algebras . . . . . 1169

Lucien Le Cam, An approximation theorem for the Poisson binomial distribution ...... 1181

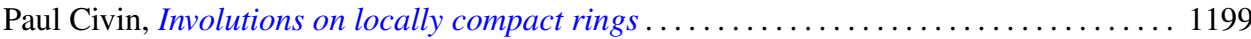

Earl A. Coddington, Normal extensions of formally normal operators . . . . . . . . . 1203

Jacob Feldman, Some classes of equivalent Gaussian processes on an interval ........ 1211

Shaul Foguel, Weak and strong convergence for Markov processes . . . . . . . . . . . 1221

Martin Fox, Some zero sum two-person games with moves in the unit interval ........ 1235

Robert Pertsch Gilbert, Singularities of three-dimensional harmonic functions . . . . . . . 1243

Branko Grünbaum, Partitions of mass-distributions and of convex bodies by

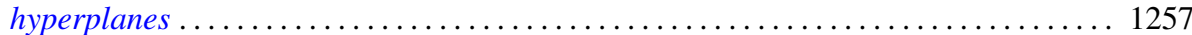

Sidney Morris Harmon, Regular covering surfaces of Riemann surfaces ........... 1263

Edwin Hewitt and Herbert S. Zuckerman, The multiplicative semigroup of integers

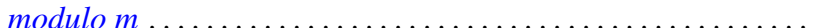

Paul Daniel Hill, Relation of a direct limit group to associated vector groups . ......... 1309

Calvin Virgil Holmes, Commutator groups of monomial groups . .

James Fredrik Jakobsen and W. R. Utz, The non-existence of expansive homeomorphisms

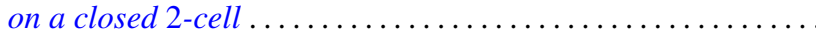

John William Jewett, Multiplication on classes of pseudo-analytic functions . . . . . . . 1323

Helmut Klingen, Analytic automorphisms of bounded symmetric complex domains . . . . 1327

Robert Jacob Koch, Ordered semigroups in partially ordered semigroups . . . . . . . . 1333

Marvin David Marcus and N. A. Khan, On a commutator result of Taussky and

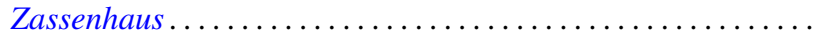

John Glen Marica and Steve Jerome Bryant, Unary algebras......

Edward Peter Merkes and W. T. Scott, On univalence of a continued fraction . . . . . . . 1361

Shu-Teh Chen Moy, Asymptotic properties of derivatives of stationary measures . . . . . 1371

John William Neuberger, Concerning boundary value problems . . . . . . . . . . . 1385

Edward C. Posner, Integral closure of differential rings . . . . . . . . . . . . . . . . . 1393

Marian Reichaw-Reichbach, Some theorems on mappings onto . . . . . . . . . . . . . 1397

Marvin Rosenblum and Harold Widom, Two extremal problems . . . . . . . . . . . . . . . . 1409

Morton Lincoln Slater and Herbert S. Wilf, A class of linear differential-difference

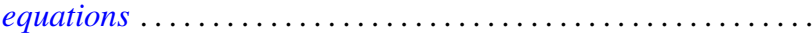

Charles Robson Storey, Jr., The structure of threads . . . . . . . . . . . . . . . . . . 1429

J. François Treves, An estimate for differential polynomials in $\partial / \partial z_{1},, \cdots, \partial / \partial z_{-} n \ldots \ldots 1447$

J. D. Weston, On the representation of operators by convolutions integrals . . . . . . . . 1453

James Victor Whittaker, Normal subgroups of some homeomorphism groups ......... 1469 